\title{
High Intensity Laser Interactions with Atomic Clusters
}

\author{
T. Ditmire
}

August 7, 2000

U.S. Department of Energy

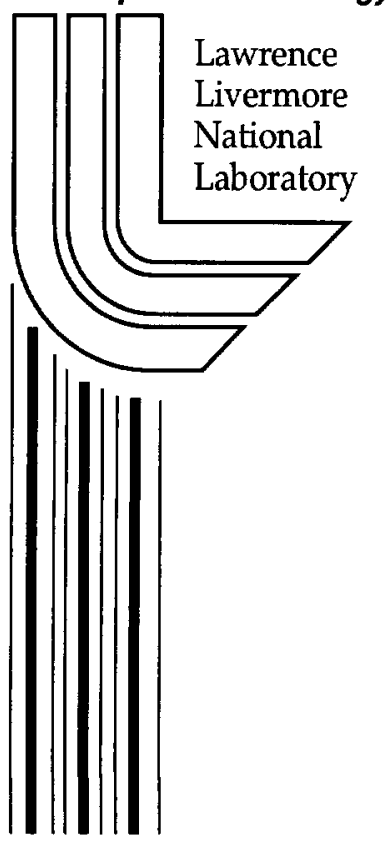




\section{DISCLAIMER}

This document was prepared as an account of work sponsored by an agency of the United States Government. Neither the United States Government nor the University of California nor any of their employees, makes any warranty, express or implied, or assumes any legal liability or responsibility for the accuracy, completeness, or usefulness of any information, apparatus, product, or process disclosed, or represents that its use would not infringe privately owned rights. Reference herein to any specific commercial product, process, or service by trade name, trademark, manufacturer, or otherwise, does not necessarily constitute or imply its endorsement, recommendation, or favoring by the United States Government or the University of California. The views and opinions of authors expressed herein do not necessarily state or reflect those of the United States Government or the University of California, and shall not be used for advertising or product endorsement purposes.

This work was performed under the auspices of the U. S. Department of Energy by the University of California, Lawrence Livermore National Laboratory under Contract No. W-7405-Eng-48.

This report has been reproduced directly from the best available copy.

Available electronically at http://www.doe.gov/bridge

Available for a processing fee to U.S. Department of Energy and its contractors in paper from

U.S. Department of Energy

Office of Scientific and Technical Information P.O. Box 62

Oak Ridge, TN 37831-0062

Telephone: (865) 576-8401

Facsimile: (865) 576-5728

E-mail: reports@adonis.osti.gov

Available for the sale to the public from

U.S. Department of Commerce

National Technical Information Service

5285 Port Royal Road

Springfield, VA 22161

Telephone: (800) 553-6847

Facsimile: (703) 605-6900

E-mail: orders@ntis.fedworld.gov

Online ordering: http://www.ntis.gov/ordering.htm

OR

Lawrence Livermore National Laboratory

Technical Information Department's Digital Library http://www.llnl.gov/tid/Library.html 


\title{
High Intensity Laser Interactions with Atomic Clusters
}

\author{
Principal Investigator: \\ Todd Ditmire \\ Laser Science and Technology, Laser Program \\ Lawrence Livermore National Laboratory \\ P. O. Box $808, L-477$ \\ Livermore, CA 94550 \\ Phone: $925-422-1349$ \\ FAX: $925-422-5537$ \\ e-mail: ditmire1 @llnl.gov
}

\section{Program Scope:}

The development of ultrashort pulse table top lasers with peak pulse powers in excess of 1 TW has permitted an access to studies of matter subject to unprecedented light intensities. Such interactions have accessed exotic regimes of multiphoton atomic and high energy-density plasma physics. Very recently, the nature of the interactions between these very high intensity laser pulses and atomic clusters of a few hundred to a few thousand atoms has come under study. Such studies have found some rather unexpected results, including the striking finding that these interactions appear to be more energetic than interactions with either single atoms or solid density plasmas. Recent experiments have shown that the explosion of such clusters upon intense irradiation can expel ions from the cluster with energies from a few keV to nearly $1 \mathrm{MeV}$. This phenomenon has recently been exploited to produce DD fusion neutrons in a gas of exploding deuterium clusters. Under this project, we have undertaken a general study of the intense femtosecond laser cluster interaction. Our goal is to understand the macroscopic and microscopic coupling between the laser and the clusters with the aim of optimizing high flux fusion neutron production from the exploding deuterium clusters or the $\mathrm{x}$-ray yield in the hot plasmas that are produced in this interaction. In particular, we are studying the physics governing the cluster explosions. The interplay between a traditional Coulomb explosion description of the cluster disassembly and a plasma-like hydrodynamic explosion is not entirely understood, particularly for small to medium sized clusters ( $<1000$ atoms) and clusters composed of low-Z atoms. We are focusing on experimental studies of the ion and electron energies resulting from such explosions through various experimental techniques. We are also examining how an intense laser pulse propagates through a dense medium containing these clusters.

\section{Recent Progress}

Much of the research undertaken this year has been focused on the interaction of our intense $30 \mathrm{fs}$ pulses with deuterium clusters. This work is motivated by our recent observation of DD fusion in a gas of laser irradiated deuterium clusters. One of the principal goals of this project is to understand the explosion mechanisms of these clusters so that they can be manipulated to enhance the fusion yield. We have also undertaken studies of third harmonic generation from these intense cluster interactions to gain understanding of the electron oscillation dynamics during the interactions. Finally, we have examined hot electron generation from quite large clusters to gain information about how the nature of

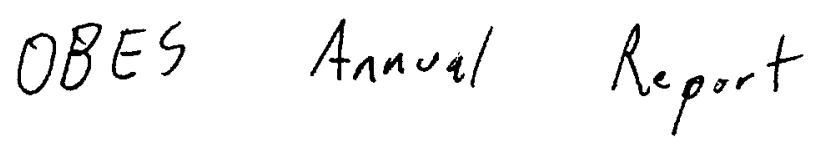


the interaction evolves from the sub wavelength scale clusters to clusters of size approaching the laser wavelength (a size scale which may also, ultimately, be interesting for fusion research).

\section{1) Interactions with deuterium clusters}

We have conducted a variety of studies on the interaction of $30 \mathrm{fs}$ pulses focused to intensity $>10^{16}$ $\mathrm{W} / \mathrm{cm}^{2}$ into gases of deuterium clusters. For example, we have conducted deuterium ion time of flight energy spectroscopy. These measurements were conducted from ions escaping the plasma in a high density gas jet (and were consequently not ideal because of space charge problems and ion slowing down in the surrounding gas. Future experiments solve these problems.) This measurement indicated that ions with energy out to $30 \mathrm{keV}$ are produced in the exploding clusters, however, detailed studies of ion energy spectra require an apparatus enabling interactions with a low density cluster beam.

One of the principal diagnostics in these experiments has been the measurement of the $2.45 \mathrm{MeV}$ fusion neutron production. To gain information on the explosion mechanisms, we examined the fusion neutron yield as a function of cluster size (see ref [1]). We find that the fusion yield increases rapidly as the average cluster size increases from 5 to $8 \mathrm{~nm}$. We vary the average cluster size by changing the temperature of the gas jet backing reservoir with liquid helium cooling. The rate of the yield increase with cluster size increase appears to be consistent with a Coulomb explosion model of the ion ejection (In this model, the laser field strips the cluster of its electrons on a time scale much faster than the cluster expansion. The Coulomb forces between ions then drive an explosion. In this simple picture, the ion energies should scale as the square of the cluster size.) In addition, we find that as we increase the average cluster size further to $10 \mathrm{~nm}$, the yield rolls over. We have conducted extensive interferometric probing of the plasmas to gain information on the laser propagation dynamics and now believe that this roll over is the result of increased laser absorption and energy depletion in the front edges of the gas jet. These results are currently under analysis and will be submitted for publication shortly.

\section{2) Two color pump probe experiments}

We have also begun pump probe experiments to explore the expansion dynamics of larger, laser heated clusters. In particular, we have constructed an experiment to examine the third harmonic generation of $800 \mathrm{~nm}$ pulses in a gas of xenon clusters as the clusters expand from the photoionization and heating of a an initial pump pulse (which has a wavelength of $400 \mathrm{~nm}$ ). This pump-probe experiment is designed to yield information on the nonlinear oscillation dynamics of the electron cloud in an expanding cluster. This experiment follows on experiments conducted at LLNL two years ago on the linear absorption of a probe laser pulse in xenon clusters as a function of delay after an ionizing pump.

We have conducted initial experiments and have seen some variation of third harmonic signal as delay between the $800 \mathrm{~nm}$ and $400 \mathrm{~nm}$ pulses. We have not yet reproduced the original absorption measurement (which used $800 \mathrm{~nm}$ pulses for both pump and probe) and attribute this to inadequate intensity in the pump pulse to drive the xenon explosion. We have procured appropriate optics to enhance the pump intensity and will begin another round of experiments in the fall.

\section{3) Interactions with large, wavelength scale clusters}

Finally, we have conducted a series of experiments on the production of fast electron during the interaction of our intense femtosecond pulses with clusters of diameter approaching that of the laser 
wavelength. In these experiments, we produced water clusters with diameter around $1 \mu \mathrm{m}$ using a special jet developed in a collaboration with Tom Donnelly at Harvey Mudd College. The 30 fs laser pulse was focused to an intensity of nearly $10^{18} \mathrm{~W} / \mathrm{cm}^{2}$ into a spray of these small water droplets (whose sizes were characterized by Mie scattering).

The laser produced hot electrons which then produced hard $\mathrm{x}$-rays via bremsstrahlung. We measured the $x$-ray spectra to gain information about the electron spectra. These measurements indicated that the laser irradiation produced electrons with an effective temperature of around $1 \mathrm{MeV}$ [3]. This result is remarkable because hot electrons produced from a planar target under nearly identical irradiation conditions exhibited a hot electron temperature only half that from the water droplets. We have compared these experimental results with particle-in-cell calculations and have found that this enhancement in hot electron temperature is a result of the laser field distribution around the wavelength scale particle. We are conducting further studies to ascertain the scaling of hot electrons with cluster size.

\section{Future Research Plans}

Our future research plans are aimed at an understanding of the interactions between the laser and single clusters to understand in more detail the energy deposition mechanisms. Studies during the previous year have concentrated on interactions of the laser pulse with high density gas jets containing clusters. By using a low density molecular beam of clusters, we intend to undertake a series of studies on single cluster interactions.

To do these future experiments we have constructed a time-of-flight spectrometer coupled to a molecular beam. We have fitted a chamber with a cryogenically cooled gas jet, capable of producing large hydrogen and deuterium clusters with sizes ranging from a 1 to $10 \mathrm{~nm}$. Laser pulses are focused with an aspheric lens into this beam of clusters and fast ejected particles are detected along an axis perpendicular to both the laser and the cluster beam. This spectrometer is designed to yield information on both ion and electron energy spectra. Ion spectra will be characterized both through direct, field free ion time of flight as well as through the use of charged retarding grids. This spectrometer will allow us to examine ions with energy up to $1 \mathrm{MeV}$ and will allow charge state differentiation on ions with energy to charge state ratios up to $20 \mathrm{keV} / \mathrm{Z}$. We will also analyze electron spectra, which will be measured by scanning the voltage on the retarding grids.

1) Our first set of experiments will be to examine the ion energy distributions from hydrogen clusters. This is important information in calculating the expected fusion yield from exploding deuterium clusters. We intend to examine these distributions as a function of average cluster size (varied by changing the gas jet temperature) as well as laser intensity, wavelength and pulse width. We will also examine electron energy distributions. This information will be compared to simulations of the laserhydrogen cluster interactions being developed by Ken Kulander now at LLNL. This round of experiments will essentially examine explosions in the pure Coulomb explosion regime from low $\mathrm{Z}$ species. Information derived here will then be used to optimize fusion yield in the high density deuterium gas jet experiments.

2) We then intend to examine spectra from higher $\mathrm{Z}$ species (namely $\mathrm{N}_{2}, \mathrm{Ar}, \mathrm{Kr}$ and $\mathrm{Xe}$ ) This will allow us to explore the nature of the cluster explosions as it evolves from a pure Coulomb explosion to a hydrodynamic explosion. Here, electron and ion spectra will be compared with particle dynamics 
simulations (i.e. the Kulander model) as well as with hydrodynamic simulations (i.e. the Hyades hydrocode).

3) Additional experiments will follow up on the two color pump probe experiments. While we intend to continue the third harmonic experiments in Xe clusters, we will also begin to look at electron and ion spectra from these two pulse interactions. Once again, we will likely concentrate on deuterium clusters with an eye toward optimizing ion energies for fusion studies.

4) Finally, we will follow up on the micron scale droplet experiments. In particular, we will likely examine the electron spectra directly. We will begin with the electrostatic TOF spectrometer, however, it is likely that we will require a magnetic spectrometer to fully characterize the fast electrons produced in these interactions.

\section{Papers published or submitted on work supported by this grant:}

1) J. Zweiback, R.A. Smith, T.E. Cowan, G. Hays, K.B. Wharton, and T. Ditmire, "Nuclear Fusion Driven by Coulomb Explosions of Large Deuterium Clusters," Phys. Rev. Lett. 84, 2634 (2000).

2) T. Ditmire, J. Zweiback, V. P. Yanovsky, T. E. Cowan, G. Hays, and K. B. Wharton, "Studies of Nuclear Fusion in Gases of Deuterium Clusters Heated by a Femtosecond Laser," Phys. Plas. 7, 1993 (2000).

3) T. D. Donnelly, M. Rust, I. Weiner, M. Allen, R. A. Smith, C. A. Steinke, S. Wilks, J. Zweiback, T. E. Cowan, and T. Ditmire, "Hard X-ray Production from Intense Laser Irradiation of WavelengthScale Particles" Phys. Rev. Lett., submitted.

4) J. Zweiback, T.E. Cowan, J. Hartley, G. Hays, R. Howell R.A. Smith, C. Steinke, and T. Ditmire, "Characterization of Fusion Burn Time in Exploding Deuterium Cluster Plasmas," Phys. Rev. Lett. submitted.

This work was performed under the auspices of the U.S. Department of Energy by the University of California, Lawrence Livermore National Laboratory under Contract No. W-7405-Eng-48. 